\title{
No Evidence for Impairment of Venous Hemodynamics in Children or Young Adults with Pediatric-Onset Multiple Sclerosis
}

\author{
S. Laughlin, C.K. Macgowan, J. Traubici, K. Chan, S. Khan, D.L. Arnold, R.A. Marrie, and B. Banwell
}

\begin{abstract}
BACKGROUND AND PURPOSE: Chronic cerebrospinal venous insufficiency is a postulated etiologic factor for multiple sclerosis, but the higher frequency with longer disease duration and progressive disability suggests that chronic cerebrospinal venous insufficiency is secondary to chronic disease. We evaluated the presence of chronic cerebrospinal venous insufficiency in pediatric-onset MS.
\end{abstract}

MATERIALS AND METHODS: Twenty-six pediatric patients with MS (18 years of age or younger), 26 age-matched healthy controls, and 13 young adults with pediatric-onset MS underwent sonography of the internal jugular, vertebral, and deep cerebral veins. Five venous hemodynamic criteria were assessed, with 2 criteria required for chronic cerebrospinal venous insufficiency. MR imaging studies, performed in the pediatric patients with MS and healthy control groups, included intracranial 2D time-of-flight MR venography and velocitysensitive phase-contrast sequences. Contrast-enhanced brain MR images were obtained in pediatric patients with MS to further evaluate venous patency. We used paired $t$ tests, Wilcoxon matched pairs, McNemar tests, and exact conditional logistic regression to estimate the association of chronic cerebrospinal venous insufficiency with MS.

RESULTS: Fifty participants (73.5\%) had normal ultrasound findings, 15 (23.1\%) met 1 venous hemodynamic criterion, and 2 pediatric patients with MS and lyoung adult with pediatric-onset MS met chronic cerebrospinal venous insufficiency criteria. Chronic cerebrospinal venous insufficiency was not associated with MS (odds ratio, 2.41; $95 \% \mathrm{Cl}$, 0.19-infinity). Demographic and disease characteristics did not differ between the patients with MS meeting chronic cerebrospinal venous insufficiency criteria $(n=3)$ and those who did not $(n=36$; all, $P>.05)$. The mean (SD) MR imaging measures of intracerebral flow did not differ between the 2 pediatric patients with MS meeting chronic cerebrospinal venous insufficiency criteria $(0.85 \pm 0.11)$ and healthy controls $(0.87 \pm 0.16, P=.50)$; no child demonstrated venous obstruction.

CONCLUSIONS: Chronic cerebrospinal venous insufficiency is rarely observed in children or young adults with pediatric-onset MS. Venous anatomy and flow rates indicate that venous outflow is intact in pediatric patients with MS. Our findings argue against chronic cerebrospinal venous insufficiency as a component of MS etiology.

ABBREVIATIONS: $A P O M S=$ young adults with pediatric-onset $\mathrm{MS} ; \mathrm{CCSVI}=$ chronic cerebrospinal venous insufficiency; $\mathrm{EV}=$ epidural vein; $\mathrm{HC}=$ healthy control; $\mathrm{IJV}=$ internal jugular vein; pedMS = pediatric patients with MS; RRMS = relapsing-remitting MS; $\mathrm{VH}=$ venous hemodynamic

C hronic cerebrospinal venous insufficiency (CCSVI), a sonography-defined pattern of cerebral venous drainage, has been reported in a variable proportion of adults with multiple sclerosis (reviewed in Awad et $\mathrm{al}^{1}$ and Laupacis et $\mathrm{al}^{2}$ ). A combination of

Received December 16, 2012; accepted after revision January 29, 2013.

From the Departments of Diagnostic Imaging (S.L., J.T.), Medical Biophysics (C.K.M., K.C.), and Neuroscience and Mental Health (S.K., B.B.), The Hospital for Sick Children and Research Institute, University of Toronto, Ontario, Canada; McConnell Brain Imaging Center (D.L.A.), McGill University, Montreal, Quebec, Canada; Departments of Internal Medicine (Neurology) and Community Health Sciences (R.A.M.), University of Manitoba, Winnipeg, Manitoba, Canada; and Department of Neurology (B.B.), Children's Hospital of Philadelphia, Philadelphia, Pennsylvania.

Statistical analysis was performed by Ruth Ann Marrie. Drs Laughlin, Banwell, Arnold, Marrie, and Macgowan contributed to the study concept and design. Drs Laughlin, Banwell, and Macgowan and S. Khan, K. Chan, and J. Traubici were instrumental in the acquisition of data. Drs Marrie, Banwell, Laughlin, and Macgowan provided data analysis and interpretation. Drs Laughlin, Banwell, findings of altered venous flow, venous stenoses with presumed secondary venous drainage patterns (termed "excision circles"), or reflux in extracranial draining veins led to the term CCSVI. ${ }^{3}$ In the original report of CCSVI in adult patients with MS, sonographic studies of the internal jugular and vertebral veins and transcranial Doppler studies of deep cerebral venous structures showed $\geq 1$ of 5 parameters that were absent in healthy subjects. ${ }^{4}$

\footnotetext{
Marrie, and Macgowan provided critical revision of the manuscript for important intellectual content. Dr Banwell was primarily responsible for study supervision and procurement of grant support.

This work was supported by grants from the Multiple Sclerosis Society of Canada. Please address correspondence to Brenda Banwell, MD, Division Chief, Neurology, Professor of Pediatrics (Neurology), Children's Hospital of Philadelphia, 34th and Civic Center Blvd, Philadelphia, PA. 19104-4399; e-mail: banwellb@email.chop.edu

三 Indicates article with supplemental on-line table

http://dx.doi.org/10.3174/ajnr.A3661
} 
These parameters included reflux in the internal jugular veins (IJVs) or vertebral veins in sitting or supine positions (in contrast to the anticipated venous flow toward the heart); spontaneous intravenous reflux ascending superiorly into at least 1 of the deep cerebral veins, including the internal cerebral veins, basal veins of Rosenthal, or vein of Galen; evidence of proximal IJV stenosis with B-mode echo color high-resolution Doppler sonography; lack of detectable venous flow on Doppler sonography in the IJVs or vertebral veins; and reversed postural control of the main cerebral venous outflow pathway. Transfemoral catheter venography was used in these studies to more fully delineate the venous anatomy. ${ }^{3}$

On the basis of these observations, it was postulated that impaired venous drainage, particularly when accompanied by reflux of flow, may lead to increased intracerebral venous pressure, which, in turn, may exacerbate transudation of inflammatory cells through the perivenous endothelial cells of the blood-brain barrier. If impaired cerebrospinal venous drainage is the fundamental pathologic underpinning of MS, then such impairment should be evident from the onset of disease. However, data showing a higher frequency of CCSVI in persons with MS who are older, ${ }^{5}$ have secondary-progressive MS, ${ }^{5}$ or have greater physical disability $^{5,6}$ suggest that CCSVI reflects chronic disease rather than being an etiologic factor as originally proposed. ${ }^{3}$

If CCSVI is an etiologic factor for MS, it should be evident even in the youngest patients with MS. Before exploring such an assertion, it is first important to establish whether pediatric-onset and adult-onset MS are comparable clinical disorders. The onset of MS during childhood or adolescence is associated with a relapsing-remitting MS (RRMS) clinical course in $>95 \%$ of patients. ${ }^{7}$ While clinical relapse features are similar in pediatric and adult patients with RRMS, the relapse rate appears higher in pediatric patients with MS, ${ }^{8}$ and very young pediatric patients with MS may experience relapses associated with encephalopathy. ${ }^{9}$ The 2010 revision of the McDonald diagnostic criteria for MS encompassed pediatric patients with $\mathrm{MS}^{10}$ and has now been validated in pediatric patients with MS ${ }^{11}$ with the caveat that very young children may have unique clinical and MR imaging features at the onset and a more typical adult-onset phenotype with time. ${ }^{12}$ Overall, clinical, biologic, and neuroimaging data all support a shared biology between pediatric- and adult-onset MS.

Patients with pediatric-onset MS provide an opportunity to evaluate the prevalence of CCSVI in a young MS population without the potential confounders of disability or age-related comorbid health conditions. While the presence of CCSVI in a pediatric MS population would support an etiologic role for CCSVI, we hypothesized that CCSVI is not a component of MS etiology and, if present, is a consequence of long-standing disease and aging vessels. We thus evaluated the frequency of CCSVI in children with MS and a group of adult patients with MS who experienced MS onset during childhood and thus have prolonged disease duration but still young vasculature. We used the originally described $^{4}$ high-resolution echo color Doppler and transcranial color-coded Doppler sonography methods. A limitation of sonographic assessment of venous patency relates to artifactual narrowing induced by pressure on the vessel by the sonographic probe, ${ }^{13}$ and we thus also evaluated venous anatomy by using phase-contrast measurements in the upper neck and 2D TOF MR venography assessment of the dural venous sinuses. Finally, abnormalities of venous anatomy or compromised venous flow (stenosis) alone do not necessarily lead to global cerebrospinal venous insufficiency or to increased venous pressures. The cerebral and neck veins are part of a low-resistance system with considerable redundancy in terms of bilaterality and with respect to the capacity for the use of collateral vessels. Partial or complete obstruction of a single vessel may have little impact on total cerebral venous outflow. We thus also measured venous blood flow pulsatility as a surrogate for elevated venous pressure. Downstream constriction of venous flow tends to shift a greater proportion of the total pressure drop between the aorta and vena cava toward these constricted vessels. The vessels upstream of the constriction thereby experience higher pressures and greater cardiac pulsation. Using these 3 methods, we provide a comprehensive assessment of cerebral venous flow in pediatric MS.

\section{MATERIALS AND METHODS}

Our primary study outcome was the frequency of CCSVI in pediatric patients with MS compared with age-matched healthy children based on sonography. We also aimed to correlate sonographic evidence of venous insufficiency in pediatric patients with MS and controls with MR venography and phase-contrast MR evaluation of venous outflow. A tertiary objective was to determine whether CCSVI was present in young adults with pediatriconset MS and, if so, whether CCSVI was associated with disease duration.

Participants. To address our primary outcome, we consecutively enrolled 2 participant groups: 1) pediatric MS: children and adolescents, 18 years of age or younger, with relapsing-remitting $\mathrm{MS}^{14}$ followed in the pediatric MS program at the Hospital for Sick Children between July 2010 and March 2012; and 2) healthy controls (HCs). All patients with RRMS registered in our MS program were notified by mail and in person during clinic visits. For each pediatric patient with MS (pedMS), an unrelated agematched healthy control participant was recruited through local advertisement, none of whom had a first degree relative with MS. To address our tertiary study objective, we also enrolled young adults with pediatric-onset MS (APOMS) previously followed in the pediatric MS program. We did not enroll healthy adult controls, given that our goal was to explore whether features of CCSVI were present in young adults with pediatric-onset RRMS, not to compare the relative frequency of such features with those of healthy adults. Furthermore, a wealth of studies has already documented the frequency of sonographic features of CCSVI in healthy adult cohorts.

All participants were required to avoid caffeine-containing fluids on the day of testing. None of the participants were receiving stimulant medications or reported exposure to illicit drugs, had a history of head or neck injury, or had known cerebrovascular disease. All patients with MS were $>30$ days from most recent relapse or corticosteroid exposure. Demographic data, age at onset, disease duration, current medications, and Expanded Disability Status Scale scores were captured for all patients with MS on the day of the sonography.

AJNR Am J Neuroradiol 34:2366-72 Dec 2013 www.ajnr.org 
All participants and their families provided informed consent, and the study was approved by the Research Ethics Board at the Hospital for Sick Children.

Sonography. Two experienced pediatric sonography technologists and 2 pediatric radiologists attended a 1-week Cerebral Venous Function and Anomaly Program at Buffalo Neuroimaging Analysis Center, The Jacobs Neurologic Institute, State University of New York, Buffalo (R. Zivadinov, MD, Program Director), which included instruction, demonstration, and hands-on training in cerebrospinal venous scanning by using similar sonographic methods previously outlined by Zamboni et al. ${ }^{3}$

Sonography technologists and pediatric radiologists were blinded to participant group. Participants were brought into the sonography suite and were positioned and draped by a study assistant before the ultrasonographer entered the room. All participants were requested to remain silent during the examination. After completing the sonography, the ultrasonographer left the room before the participant was permitted to move from the sonography table. All ultrasounds were observed by a study assistant to ensure compliance with blinding procedures. Because we did not match the APOMS group to controls, we cannot exclude the possibility that the sonography staff may have been unblinded to MS diagnosis in these older participants (all 23 years of age or younger).

Sonographic examinations were performed with participants positioned supine and at $90^{\circ}$ (sitting) following 2 minutes of rest and normal breathing in each position by using an IU 22 sonography unit (Philips Medical Systems, Bothell, Washington). For studies of the neck, a high-frequency L9-3-MHz linear transducer or a $\mathrm{C} 8-5-\mathrm{MHz}$ small curved-array transducer was used to assess the internal jugular veins and vertebral veins bilaterally, applying minimal neck pressure. For the transcranial studies, a lower frequency probe was used (S5-1 transcranial sector probe) to assess either a basal vein of Rosenthal or internal cerebral vein. Sonographic data were scored by consensus according to the 5 venous hemodynamic $(\mathrm{VH})$ criteria detailed by Zamboni et al. ${ }^{3}$ CCSVI was considered present if 2 of the VH criteria were met (as detailed in the On-line Table).

MR Imaging Studies. As per our primary study objective, pedMS and $\mathrm{HC}$ groups were also offered noninvasive MR imaging assessment of the brain and intracranial venous anatomy, including 2D time-of-flight MR venography and phase-contrast MR imaging measures of venous flow interrogating overall cerebral blood circulation by using a standardized MR imaging protocol on the same 3T scanner (Magnetom TrioTim; Siemens, Erlangen, Germany), while contrast-enhanced MR imaging by using intravenous gadolinium was acquired in pedMS only (On-line Table). The TOF-MR venography contrast-enhanced MR imaging evaluated the patency of the jugular bulbs, dural venous sinuses, and internal cerebral veins.

Phase-Contrast MR Imaging. Axial-oblique phase-contrast MR imaging measures of venous flow data were acquired from the neck with the section oriented perpendicular to the dominant vessel orientations at the C2-C3 level. Participants were imaged supine during free breathing to maintain resting cardiovascular physiology. A segmented and cardiac-gated 2D phase-contrast
MR imaging sequence with through-plane velocity encoding was used (parameters listed in the On-line Table). Flow images were acquired at 2 velocity sensitivities, the first to measure right and left IJVs, internal carotid artery, and vertebral artery flows (maximum velocity without aliasing $=100 \mathrm{~cm} / \mathrm{s}$ ) and the second to measure slower right and left epidural vein (EV) flows (maximum velocity without aliasing $=30 \mathrm{~cm} / \mathrm{s}$ ). If aliasing was detected, the velocity sensitivity of the acquisition was reduced and the scan was repeated. The scan time for each series was approximately 2 minutes. To ensure that intraindividual flow values were stable, we acquired these sequences twice, within the first 10 minutes of imaging and just before completion of the MR imaging examination. The 2 flow values for each participant were compared for each of the 8 vessels.

For each vessel, total flow per minute (milliliter/min) was calculated by using the analysis package Segment (Version 1.8; Medviso, Lund, Sweden). ${ }^{15}$ This validated software provided automatic detection of the vessel lumen throughout the cardiac cycle, based on an initial manual contour prescribed by the user, and enabled cinematic playback of the region of interest superimposed on the images to verify placement. Background phase correction was also applied on the basis of surrounding static tissue. ${ }^{16}$ Region-of-interest assessment was performed independently by 2 investigators (K.C., C.K.M.). Data from a subset of subjects were analyzed twice by both investigators to test inter- and intraobserver reliability according to the intraclass correlation coefficient on a per-vessel basis.

If flow in a specific vessel could not be determined (vessel not visualized, aliasing of flow, artifacts) at both time points, the net flow rate for that participant was not calculated. To detect the potential redistribution of venous flow secondary to venous stenoses, we calculated the ratio of total measured venous flow to total measured arterial flow, (IJV + EV) / (Internal Carotid + Vertebral Artery). We hypothesized that this ratio would be lower in participants with venous insufficiency secondary to flow redistribution to alternative venous pathways. ${ }^{17,18}$

\section{Statistical Analysis}

Continuous variables were summarized as mean (SD) or median (interquartile range) as appropriate. Categoric variables were described as frequency (percentage). Continuous variables were compared between the pedMS and HC groups by using a paired $t$ test or Wilcoxon matched pairs test as appropriate. Categoric variables were compared by using the McNemar test. Exact conditional logistic regression was used to examine the association of CCSVI with pediatric MS among the pedMS and matched HCs; the number of affected individuals was too small to adjust for factors that were not matched. In the MS group, we evaluated sonographic findings as a function of time by comparing the disease duration between MS cases with and without CCSVI (as defined by the presence of 2 of the $5 \mathrm{VH}$ criteria) by using a Wilcoxon test. Furthermore, we correlated disease duration with the total number of positive VH criteria by using a Spearman rank correlation. The mean and SD were calculated for the venous-toarterial flow ratio for all pedMS and HCs, provided that values for all 8 vessels were recorded on at least 1 of the 2 phase-contrast-MR imaging time points. For paired comparisons, both 
members of the matched pair were required to have results available for all 8 vessels. $P$ values $<.05$ were considered significant. Analyses were performed by using SAS, Version 9.2 (SAS Institute, Cary, North Carolina).

\section{RESULTS}

Participants. Table 1 details the demographic features of the 65 (26 pedMS, $26 \mathrm{HCs}$, and 13 APOMS) participants. Most patients with MS were receiving disease-modifying therapy; all were considered to have RRMS and all had normal ambulation.

Sonography. Sonographic study findings were completely normal, with no evidence for any of the $5 \mathrm{VH}$ criteria in 47 of 65 participants (72.3\%). Eighteen participants demonstrated 1 or 2 of the VH criteria (Table 2). VH criterion 3 was the most common finding, present in 14 of 65 participants (21.5\%), while 6 participants met VH criterion 1 (9.2\%) and 1 participant met VH criterion $5(1.5 \%)$. None of the participants met VH criterion 2 or 4. The frequency of each criterion across the 3 participant groups is also detailed in Table 2. In an unmatched analysis of the pedMS and HC groups, the frequency of meeting any one criterion did not differ between groups (Fisher exact test; all, $P>.05$ ); findings were the same by using a matched analysis.

CCSVI criteria were met in 3 participants, 2 pedMS and 1 APOMS. All 3 participants met CCSVI criteria based on positive findings for criterion 1 and criterion 3. Using exact conditional logistic regression including the matched pedMS and HCs, we

Table 1: Demographic and clinical features of the 65 participants

\begin{tabular}{lccc}
\multicolumn{1}{c}{ Feature } & $\begin{array}{c}\text { Healthy Pediatric } \\
\text { Controls }(\boldsymbol{n}=\mathbf{2 6})\end{array}$ & $\begin{array}{c}\text { Pediatric RRMS } \\
(\boldsymbol{n}=\mathbf{2 6})\end{array}$ & $\begin{array}{c}\text { APOMS RRMS } \\
(\boldsymbol{n}=\mathbf{1 3})\end{array}$ \\
\hline Female (No.) & $18(69.2 \%)$ & $16(61.5 \%)$ & $8(61.5 \%)$ \\
Age at enrollment (yr) (mean) (SD) & $15.4(3.6)$ & $16.2(2.5)$ & $20.3(2.9)$ \\
Range (yr) & $(8.94-18.61)$ & $(9.36-18.08)$ & $(18.24-23.76)$ \\
Age at first attack (mean) (SD) (yr) & N/A & $12.3(3.9)$ & $13.9(3.6)$ \\
Disease duration (mean) (SD) (yr) & N/A & $3.9(4.4)$ & $6.5(3.7)$ \\
$\quad$ Range (yr) & & $(0.67-13.34)$ & $(4.01-10.42)$ \\
EDSS (median, IQR) & N/A & $1.5(1.0,2.5)$ & $1.5(1.0,2.0)$ \\
Medications used (No.) (\%) & N/A & & \\
None & & $9(34.7)$ & $2(15.4)$ \\
IFN & & $11(42.3)$ & $7(53.9)$ \\
GA & & $4(15.4)$ & $2(15.4)$ \\
Other & & $2(7.7)$ & $2(15.4)$ \\
\hline
\end{tabular}

Note:-N/A indicates not applicable; EDSS, Expanded Disability Status Scale; IQR, interquartile range; IFN, interferon; GA, glatiramer acetate. found that the frequency of CCSVI did not differ between groups (odds ratio, 2.41; 95\% CI, 0.19- infinity). Unconditional logistic regression adding the APOMS group produced similar findings (odds ratio, 2.60; 95\% CI, 0.27-infinity). The 3 patients with MS meeting the criteria for CCSVI did not differ from the 36 who did not in terms of sex, average age of onset, disease duration, or Expanded Disability Status Scales score (data not shown).

MR Venography. On the TOF-MR venography (pedMS and HC) and postgadolinium sagittal T1 3D MPRAGE sequences (pedMS only), no MR imaging evidence of acute dural venous sinus thrombosis was noted. Some participants had dominant right or left transverse and/or sigmoid sinuses, while in others, these were codominant. Expected anatomic variants were also noted, including arachnoid granulations, accessory occipital sinuses, partial duplication of the superior sagittal sinus, and transosseous emissary veins. One patient with pediatric MS had a hypoplastic straight sinus, which had also been noted on previous clinical MR imaging and was stable. In addition to the MR venography, our protocol also included a sagittal postcontrast T1 3D MPRAGE sequence, which was reviewed carefully in all patients to confirm patency of the dural venous sinuses.

Phase-Contrast Vascular Flow. The mean venous-to-arterial flow ratio $(1 / \mathrm{min})$ did not differ between pedMS and HCs, being on average $0.85 \pm 0.11$ in the pedMS population and $0.87 \pm 0.16$ in HCs $(t=-0.69, P=.50)$. The mean flow ratio in the 2 pedMS who met the CCSVI criteria was $0.94 \pm 0.12$, which did not differ from the ratio in the 39 pedMS and HCs who did not meet the CCSVI criteria $(0.86 \pm 0.14, P=.49)$. Thus no difference in venous-to-arterial flow ratios was detected in subjects meeting CCSVI criteria.

Consistent identification of the internal jugular veins was possible by using not only vessel area and position but also flow direction (head-to-foot). Inter- and intraobserver agreement between flow measurements was excellent: The intraobserver intraclass correlation coefficient was $>0.97$, and the interobserver intraclass correlation coefficient

Table 2: Ultrasound findings for adults and children with MS and HCs

\begin{tabular}{|c|c|c|c|c|c|c|}
\hline \multirow[b]{2}{*}{ Group } & \multicolumn{5}{|c|}{ Venous Hemodynamic Insufficiency Criteria } & \multirow[b]{2}{*}{ CCSVI } \\
\hline & 1 & 2 & 3 & 4 & 5 & \\
\hline & $\begin{array}{l}\text { Reflux in neck } \\
\text { vessels }\end{array}$ & $\begin{array}{l}\text { Reflux in deep } \\
\text { cerebral veins }\end{array}$ & $\begin{array}{l}\text { IJV stenosis, } \\
\text { noncompliance and } \\
\text { B-mode anomalies }\end{array}$ & $\begin{array}{l}\text { Absence of } \\
\text { Doppler flow }\end{array}$ & $\begin{array}{l}\text { Negative IJV } \\
\text { CSA }\end{array}$ & $\begin{array}{l}\text { Minimum of } 2 \\
\text { criteria }\end{array}$ \\
\hline & IJV supine & $\mathrm{ICV}$ & $\mathrm{CSA}<0.3 \mathrm{~cm}^{2}$ & IJV & & \\
\hline & IJV $90^{\circ}$ & $\begin{array}{r}\text { Basal vein of } \\
\text { Rosenthal }\end{array}$ & & VV & & \\
\hline & $\begin{array}{l}\text { VV supine } \\
\text { VV } 90^{\circ}\end{array}$ & & & & & \\
\hline $\operatorname{PedMS}(n=26)$ & $3(11.5 \%)$ & 0 & $7(26.9 \%)$ & 0 & $1(3.8 \%)$ & $2(7.7 \%)$ \\
\hline $\operatorname{APOMS}(n=13)$ & $2(15.4 \%)$ & 0 & $2(15.4 \%)$ & 0 & 0 & $1(7.7 \%)$ \\
\hline $\mathrm{HCs}(n=26)$ & $1(3.8 \%)$ & 0 & $5(19.2 \%)$ & 0 & 0 & 0 \\
\hline
\end{tabular}




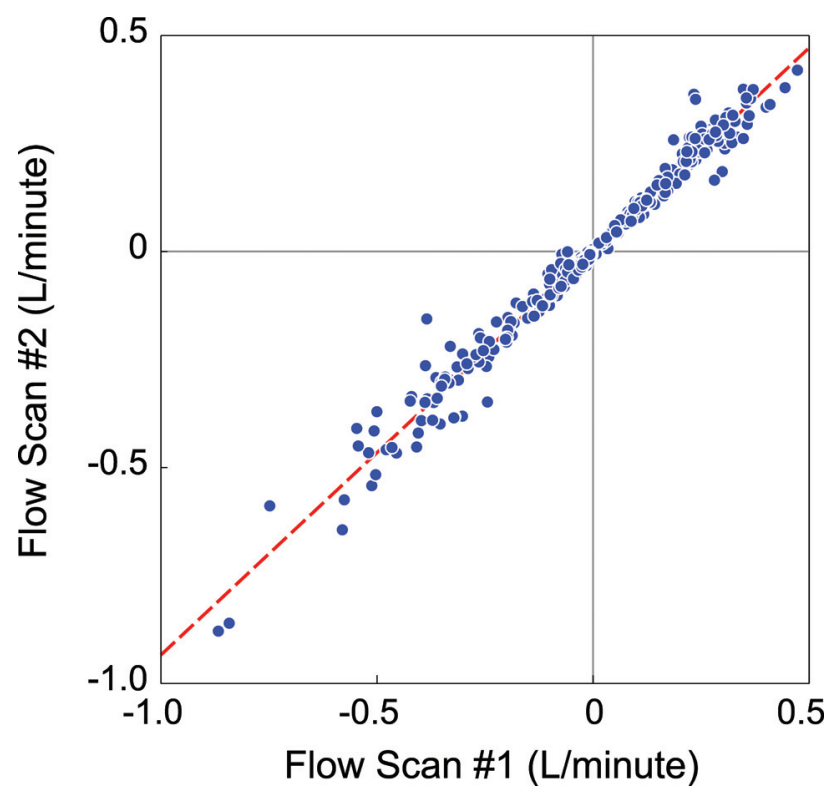

FIG 1. Reproducibility of vessel flow measurements on phase-contrast MR imaging between first and second measurements (across all vessels examined).

Table 3: Average (SD) flow rates (L/min) in arteries and veins ${ }^{\mathrm{a}}$

\begin{tabular}{lccc}
\hline \multicolumn{1}{c}{ Vessel } & pedMS & HCs & $P$ Value \\
\hline ICA, right & $0.26(0.058)$ & $0.26(0.033)$ & .63 \\
ICA, left & $0.26(0.058)$ & $0.27(0.038)$ & .92 \\
IJV, right & $-0.33(0.18)$ & $-0.33(0.21)$ & .20 \\
IJV, left & $-0.19(0.13)$ & $-0.25(0.15)$ & .19 \\
VA, right & $0.096(0.05)$ & $0.11(0.033)$ & .14 \\
VA, left & $0.10(0.037)$ & $0.12(0.045)$ & .30 \\
EVs, right & $-0.033(0.029)$ & $-0.054(0.047)$ & .066 \\
EVs, left & $-0.037(0.036)$ & $-0.050(0.030)$ & .056 \\
\hline
\end{tabular}

Note:-VA indicates vertebral artery.

${ }^{a}$ Negative values represent flow in the superior-to-inferior direction.

was $>0.93$, indicating that inherent flow differences between subjects exceeded the variance of the measurement process itself. Arterial flows were obtained in all participants in at least 1 time point. In a small number of vessels, venous flows were not obtained at either time point as follows: 3 right IJVs, 1 left IJV, 1 left EVs, and 2 right EVs. These were the result of aliasing in 2 right IJVs and poor vessel visualization in the remaining cases.

Flows measured in individual vessels were highly reproducible between the start and end of the MR imaging examination (including all vessels, $r=0.94$; 95\% CI, 0.92-0.95; Fig 1). However, absolute flows in a given vessel differed among individuals, particularly in the veins. For example, as shown in Table 3, the coefficients of variation in the right and left IJVs of HCs were $64 \%$ and $60 \%$, respectively. This finding underscores the difficulty of attributing hemodynamic significance to individual venous flows at a particular location within the complex vascular network.

\section{DISCUSSION}

In the original description of CCSVI, the $100 \%$ concordance with MS and its absence in healthy controls led to postulates that CCSVI might be congenital and etiologic in MS. ${ }^{4} \mathrm{We}$ demonstrate that CCSVI is not a key feature of pediatric MS, arguing against impaired venous drainage playing a role in the etiology of relaps- ing-remitting MS. We also failed to demonstrate a relationship between CCSVI and prolonged disease duration in a group of young adult patients with pediatric-onset MS, suggesting that CCSVI is not an obligate feature of chronic MS, at least in patients without other comorbidities or aging vasculature. Finally, we demonstrated no difference in venous pulsatility in patients with MS compared with healthy controls, arguing against the concept of raised venous pressure in MS.

When comparing the sonographic features of the 26 pediatric patients with RRMS in our study with the 35 patients with RRMS originally described by Zamboni et $\mathrm{al},{ }^{3}$ several contrasts are notable. More than $50 \%$ of the adult patients with RRMS met criterion 2 , and $>60 \%$ met criterion 4 , compared with none of our patients. Furthermore, $50 \%-77 \%$ of adult patients with RRMS met criterion 1 or 5 , while only 4 children $(7.7 \%)$ met either of these criteria, including 4 pedMS and $1 \mathrm{HC}$. However, criterion 3 was met by $26 \%$ of the adult patients with RRMS and 7 (26\%) of our pediatric patients with RRMS. Here, the contrast between our work and that of Zamboni et $\mathrm{al}^{3}$ relates to the frequency of criterion 3 detection in controls. We detected criterion 3 in 5 of 26 (19\%) healthy children, no different from its detection in our pediatric RRMS group. Zamboni et al reported that only 1 of 235 controls met criterion 3. While criterion 3 emerged as the most common sonographic finding in our study, we could not detect any evidence of dural venous sinus thrombosis above the jugular bulbs by MR venography, nor did our phase-contrast venous flow studies suggest IJV flow impairment at the level of C2. Thus, we question whether the sonographic findings have physiologic relevance. With respect to the frequency of meeting the diagnostic requirements for CCSVI, only 2 of our pedMS (7.7\%) met the criteria for CCSVI, in contrast to $100 \%$ of the adult patients with MS previously reported. ${ }^{4}$

Our study adds to the growing number of studies that have not replicated a high prevalence of CCSVI in RRMS. ${ }^{19-23}$ In the largest venous sonography study to date, $56 \%$ of adult patients with MS met the criteria for CCSVI, compared with $42 \%$ of patients with other neurologic disorders and $22 \%$ of healthy adult controls. ${ }^{24}$ In 2011, a meta-analysis supported a higher prevalence of sonographic features of CCSVI in MS compared with controls, but it highlighted numerous methodologic challenges, including a lack of blinding in some studies. ${ }^{2}$ We used a stringent blinding procedure, which ensured that the sonography technicians and radiologists were unaware of disease status. Our sonographic findings are similar to those reported in an Italian pediatric MS cohort, ${ }^{25}$ in which CCSVI was diagnosed (by consensus of 2 ultrasonographers) in 4 of 15 (26\%) pedMS and $3(18.8 \%)$ HCs. Pooling the data from both studies, which used similar sonographic techniques, yields a frequency of CCSVI of $17 \%$ (7 of 41 ) in pediatric patients with RRMS versus 7\% (3 of 42) in age-matched healthy controls $(P=.11)$. Although the total number of pediatric patients with MS evaluated to date remains low, the number studied was sufficient to address our original power calculations, which were based on the $100 \%$ frequency of CCSVI in adult-onset MS initially reported. ${ }^{4}$ As mentioned above, more recent analyses have indicated either no relationship or a much lower prevalence of CCSVI in some adult MS cohorts (summarized in Laupacis et 
$\mathrm{al}^{2}$ ). As such, our sample size is not sufficiently large to exclude the possibility of a rare association of CCSVI in pediatric MS.

Our work adds to the work of Amato et $\mathrm{al}^{25}$ by not only failing to find a relationship between CCSVI and pediatric MS but also providing evidence of normal venous pulsatility in children with MS. If raised venous pressure was an etiologic component of MS, even in the absence of visualized alteration in venous flow, then we would have expected to detect this in our pediatric patients with MS.

We hypothesized that if CCSVI were a feature of pediatric MS, then venous insufficiency should be identifiable by using quantitative methods. Zamboni et $\mathrm{al}^{3}$ have proposed that CCSVI results in venous congestion and increased intracerebral venous pressure and ultimately in $\mathrm{MS}^{26}$ As expected, absolute arterial and venous flow rates varied among individuals owing to heart rate, body mass index, and blood pressure differences. The measured arterial inflow was highly comparable with measurable venous outflow (Table 3), and flow measures were highly reproducible within individuals (Fig 1). The venous-arterial flow ratios we measured are consistent with prior reports in adults (0.85). ${ }^{17}$ Finally, venous-to-arterial flow ratios were similar in participants who did and did not meet CCSVI criteria; this finding suggests unimpaired venous outflow in the interrogated vessels of the subjects with CCSVI. This finding is consistent with the MR imaging observations that did not identify dural venous sinus thrombosis in either of the CCSVI-positive pedMS.

Some investigators have suggested that CCSVI is a consequence of chronic CNS illness, because of the higher frequency of CCSVI in patients with MS with longer disease duration and in those with more severe disability ${ }^{5}$ and the low rate of CCSVI $(18 \%)$ in adults with a clinically isolated syndrome. ${ }^{27-29}$ In such patient populations, it is difficult to disentangle the effects of long disease duration, disability, increasing age of the cerebral vasculature, and comorbid health issues. ${ }^{30}$ Therefore, we evaluated 13 young adults with pediatric-onset MS, a population with longer disease duration, little disability, no comorbidities, and young cerebral vasculature. Despite an average disease duration of 6.5 years, only 1 patient met the criteria for CCSVI and only 2 others had any abnormal $\mathrm{VH}$ parameters.

The plausibility of CCSVI as a component of MS pathobiology is further challenged by a recently published study using IJV ligation in a murine model. ${ }^{31}$ Cerebral imaging and pathologic analyses were compared among mice undergoing ligation of the IJV, sham-operated animals, and mice induced to develop experimental allergic encephalomyelitis, an animal model of neuroinflammation similar to MS. The IJV-ligated mice developed collateral venous drainage but did not develop impaired blood-brain barriers or CNS inflammation, even with bilateral IJV ligation.

\section{CONCLUSIONS}

We demonstrate that CCSVI is not a component of pediatric MS and thus is unlikely to be an etiologic component of MS. The controversy surrounding CCSVI was fueled by a powerful patient lobby for engagement, which extended to the pediatric MS population. Our study addressed our research aims and met the needs of our pediatric MS community. Many of the participants and their families commented that the ability to participate in a re- search program evaluating a new theory for MS was empowering. Our ability to reassure our patients that CCSVI is not a key component of pediatric MS will help ensure that persons with pediatric-onset MS are not subjected to unwarranted procedures.

\section{ACKNOWLEDGMENTS}

The authors thank A. Mohanta and J. Jarrin (senior sonography technologists); T. Rayner and R. Weiss (research MR imaging technologists); and. R. Zivadinov, MD, and K. Marr for CCSVI training of sonography staff. We are indebted to the children and their families for their commitment to this study.

Disclosures: Suzanne Laughlin—RELATED: Grant: Multiple Sclerosis Society of Canada and the Canadian Multiple Sclerosis Foundation, UNRELATED: No disclosures. Christopher K. Macgowan-RELATED: Grant: Multiple Sclerosis Society of Canada and the Canadian Multiple Sclerosis Foundation, UNRELATED: Dr. Macgowan receives research funding from the Canadian Institutes of Health Research, the Ontario Research and Development Fund, and the Multiple Sclerosis Society of Canada. Jeffrey Traubici has no disclosures. Stephanie Khan-RELATED: Grant: Multiple Sclerosis Society of Canada and the Canadian Multiple Sclerosis Foundation, UNRELATED: No disclosures. Douglas L. Arnold—RELATED: Grant: Multiple Sclerosis Society of Canada and the Canadian Multiple Sclerosis Foundation, UNRELATED: Consultancy: Bayer Healthcare, Biogen Idec, EMD Serono, Genentech, Genzyme, Glaxo-SmithKline, NeuroRx Research, Novartis, Roche, Merck Serono, Teva, Employment: McGill University and NeuroRx Research, Grants/Grants Pending: CIHR, MSSC, Bayer Healthcare, Patents (planned, pending or issued): Arnold et al, US Patent No. 6,347,239, Comments: patent applies to a technology for MR spectroscopy (no relevance to current article), Stock/Stock Options: NeuroRx Research. Ruth Ann Marrie-RELATED: Grant: Multiple Sclerosis Society of Canada and the Canadian Multiple Sclerosis Foundation, UNRELATED: Ruth Ann Marrie receives research funding from the Canadian Institutes of Health Research, Public Health Agency of Canada, Manitoba Health Research Council, Health Sciences Centre Foundation, Multiple Sclerosis Society of Canada. Multiple Sclerosis Scientific Foundation, Rx \&D Health Research Foundation and has conducted clinical trials funded by Bayer Inc and Sanofi-Aventis. Brenda Banwell—RELATED: Grant: Multiple Sclerosis Society of Canada and the Canadian Multiple Sclerosis Foundation, UNRELATED: Board Membership: Biogen-IDEC Advisory Board on fetal outcome from pregnancy, Comments: no financial reimbursement, Grants/Grants Pending: Multiple Sclerosis Society, Canadian Institutes of Health,* Multiple Sclerosis Scientific Research Foundation,* Dairy Farmers of Ontario,* Payment for Lectures (including service on Speakers Bureaus): Serono, Biogen-Idec, Novartis.

\section{REFERENCES}

1. Awad AM, Marder E, Milo R, et al. Multiple sclerosis and chronic cerebrospinal venous insufficiency: a critical review. Ther Adv Neurol Disord 2011;4:231-35

2. Laupacis A, Lillie E, Dueck A, et al. Association between chronic cerebrospinal venous insufficiency and multiple sclerosis: a metaanalysis. CMAJ 2011;183:E1203-12

3. Zamboni P, Galeotti R, Menegatti E, et al. Chronic cerebrospinal venous insufficiency in patients with multiple sclerosis. J Neurol Neurosurg Psychiatry 2009;80:392-99

4. Zamboni P, Menegatti E, Galeotti R, et al. The value of cerebral Doppler venous haemodynamics in the assessment of multiple sclerosis. J Neurol Sci 2009;282:21-27

5. Weinstock-Guttman B, Ramanathan M, Marr K, et al. Clinical correlates of chronic cerebrospinal venous insufficiency in multiple sclerosis. BMC Neurol 2012;12:26

6. Patti F, Nicoletti A, Leone C, et al. Multiple sclerosis and CCSVI: a population-based case control study. PLoS One 2012;7:e41227

7. Banwell B, Krupp L, Kennedy J, et al. Clinical features and viral serologies in children with multiple sclerosis: a multinational observational study. Lancet Neurol 2007;6:773-81

8. Gorman M, Healy B, Polgar-Turcsanyi M, et al. Increased relapse rate in pediatric-onset compared with adult-onset multiple sclerosis. Arch Neurol 2009;66:54-59

9. Mikaeloff Y, Caridade G, Husson B, et al. Acute disseminated en- 
cephalomyelitis cohort study: prognostic factors for relapse. Eur J Paediatr Neurol 2007;11:90-95

10. Polman CH, Reingold SC, Banwell B, et al. Diagnostic criteria for multiple sclerosis: 2010 revisions to the McDonald criteria. Ann Neurol 2011;69:292-302

11. Sadaka Y, Verhey LH, Shroff MM, et al. 2010 McDonald criteria for diagnosing pediatric multiple sclerosis. Ann Neurol 2012;72:211-23

12. Chabas D, Castillo-Trivino T, Mowry EM, et al. Vanishing MS T2bright lesions before puberty: a distinct MRI phenotype? Neurology 2008;71:1090-93

13. Doepp F, Wurfel JT, Pfueller CF, et al. Venous drainage in multiple sclerosis: a combined MRI and ultrasound study. Neurology 2011;77:1745-51

14. Polman CH, Reingold SC, Edan G, et al. Diagnostic criteria for multiple sclerosis: 2005 revisions to the "McDonald Criteria." Ann Neurol 2005;58:840-46

15. Heiberg E, Sjogren J, Ugander M, et al. Design and validation of Segment-freely available software for cardiovascular image analysis. BMC Med Imaging 2010;10:1

16. Walker PG, Cranney GB, Scheidegger MB, et al. Semiautomated method for noise reduction and background phase error correction in MR phase velocity data. J Magn Reson Imaging 1993;3:521-30

17. Stoquart-Elsankari S, Lehmann P, Villette A, et al. A phase-contrast MRI study of physiologic cerebral venous flow. J Cereb Blood Flow Metab 2009;29:1208-15

18. Haacke EM, Feng W, Utriainen D, et al. Patients with multiple sclerosis with structural venous abnormalities on MR imaging exhibit an abnormal flow distribution of the internal jugular veins. $J$ Vasc Interv Radiol 2012;23:60-68.e1-3

19. Doepp F, Paul F, Valdueza JM, et al. No cerebrocervical venous congestion in patients with multiple sclerosis. Ann Neurol 2010;68:173-83

20. Auriel E, Karni A, Bornstein NM, et al. Extra-cranial venous flow in patients with multiple sclerosis. J Neurol Sci 2011;309:102-04

21. Baracchini C, Valdueza JM, Del Sette M, et al. CCSVI and MS: a statement from the European Society of Neurosonology and Cerebral Hemodynamics. J Neurol 2012;259:2585-89

22. Sundström $P$, Wahlin A, Ambarki $K$, et al. Venous and cerebrospinal fluid flow in multiple sclerosis: a case-control study. Ann Neurol 2010;68:255-59

23. Mayer CA, Pfeilschifter W, Lorenz MW, et al. The perfect crime? CCSVI not leaving a trace in MS. J Neurol Neurosurg Psychiatry 2011;82:436-40

24. Zivadinov R, Marr K, Cutter G, et al. Prevalence, sensitivity, and specificity of chronic cerebrospinal venous insufficiency in MS. Neurology 2011;77:138-44

25. Amato M, Saia V, Hakiki B, et al. No association between chronic cerebrospinal venous insufficiency and pediatric-onset multiple sclerosis. Mult Scler 2012;18:1791-96

26. Singh AV, Zamboni P. Anomalous venous blood flow and iron deposition in multiple sclerosis. J Cereb Blood Flow Metab 2009;29:1867-78

27. Baracchini C, Perini P, Calabrese M, et al. No evidence of chronic cerebrospinal venous insufficiency at multiple sclerosis onset. Ann Neurol 2011;69:90-99

28. Blinkenberg M, Akeson P, Sillesen H, et al. Chronic cerebrospinal venous insufficiency and venous stenoses in multiple sclerosis. Acta Neurol Scand 2012;126:421-27

29. Yamout B, Herlopian A, Issa Z, et al. Extracranial venous stenosis is an unlikely cause of multiple sclerosis. Mult Scler 2010;16:1341-48

30. Dolic K, Weinstock-Guttman B, Marr K, et al. Risk factors for chronic cerebrospinal venous insufficiency (CCSVI) in a large cohort of volunteers. PLoS One 2011;6:e28062

31. Atkinson W, Forghani R, Wojtkiewicz GR, et al. Ligation of the jugular veins does not result in brain inflammation or demyelination in mice. PLoS One 2012;7:e33671

32. Zamboni P, Galeotti R, Menegatti E, et al. A prospective open-label study of endovascular treatment of chronic cerebrospinal venous insufficiency. J Vasc Surg 2009;50:1348-58.e1 